\title{
Structure-Activity Relationship of Arg10-Teixobactin: A Recently Discovered Antimicrobial Peptide ${ }^{\dagger}$
}

\author{
Fernando Albericio 1,2,3,5, Shimaa A. H. Abdel Monaim ${ }^{4}$, Yahya E. Jad ${ }^{4}$, Gerardo A. H. Acosta ${ }^{2,3}$, \\ Estelle J. Ramchuran ${ }^{4}$, Ayman El-Faham ${ }^{5}$, Thavendran Govender ${ }^{4}$, Hendrik G. Kruger ${ }^{4}$ and \\ Beatriz G. de la Torre ${ }^{6, *}$ \\ 1 School of Chemistry and Physics, University of KwaZulu-Natal, 4001 Durban, South Africa; \\ albericio@ukzn.ac.za \\ 2 Department of Organic Chemistry, University of Barcelona, 08028 Barcelona, Spain; gerardoacosta@ub.edu \\ 3 CIBER-BBN, Networking Centre on Bioengineering, Biomaterials and Nanomedicine, Barcelona Science \\ Park, 08028-Barcelona, Spain \\ 4 School of Health Sciences, University of KwaZulu-Natal, 4001 Durban, South Africa; \\ shimaa.amin10@yahoo.com (S.A.H.A.M.); yahyajad@yahoo.com (Y.E.J.); Ramchurane@ukzn.ac.z (E.J.R.); \\ Govenderthav@ukzn.ac.za (T.G.); kruger@ukzn.ac.za (H.G.K.) \\ 5 Department of Chemistry, College of Science, King Saud University, Riyadh 11451, Saudi Arabia; \\ aymanel_faham@hotmail.com \\ 6 School of Laboratory of Medicine and Medical Sciences, University of KwaZulu-Natal, 4001 Durban, \\ South Africa \\ * Correspondence: garciadelatorreb@ukzn.ac.za \\ + Presented at the 1st Molecules Medicinal Chemistry Symposium, Barcelona, Spain, 8 September 2017. \\ Published: 18 October 2017
}

The emergence of multidrug resistant bacteria has a direct impact on global public health due to the reduced potency of existing antibiotics against pathogens [1]. Hence, there is a pressing need for new drugs with different modes of action that can kill microorganisms. Antimicrobial peptides (AMPs) can be regarded as an alternative tool for this purpose since they are proven to have therapeutic effects with broad-spectrum activities [2].

In this regard, Teixobactin is a recently discovered antimicrobial cylcodepsipeptide with good activity against Gram-positive bacteria but not Gram-negative bacteria [3]. Teixobactin has been isolated from Eleftheria terrae, a non-culturable Gram-negative bacteria that belongs to the class of beta-proteobacteria. Teixobactin is the first new antibiotic to be discovered for several decades, attracting attention not only for its great activity against Gram-positive bacteria and mycobacterium tuberculosis. Teixobactin is an 11-mer peptide containing a cyclotetradepsipeptide unit in its structure. It contains five unnatural amino acid residues: D-NMe-Phe, D-Gln, D-allo-Ile, D-Thr and L-allo-enduracididine.

Recently, taking the Arg 10 -Teixobactin as a base-published by our group at the end of 2015 [4]-where the Arg substituted the non-proteinoenic residue L-allo-enduracididine, Lys scanning allowed us to identify the importance of keeping the balance between the hydrophilic and hydrophobic amino acids for the antimicrobial activity of this peptide family. Thus, the substitution of the four Ile present in the natural sequence by Lys led to a total loss of activity. On the other hand, the substitution of the polar non-charged residues and the Ala by Lys allowed the antimicrobial activity to be kept and in some cases improved [5].

Herein, the latest results in the structure-activity relationship of $\operatorname{Arg}_{10}$-Teixobactin will be discussed as well as some insights regarding the mode of action of this intriguing compound.

Acknowledgments: This work was funded in part by the following: National Research Foundation (NRF) and the University of KwaZulu-Natal (South Africa); the International Scientific Partnership Program ISPP at King Saud University (ISPP\# 0061) (Saudi Arabia); and MINECO (CTQ2015-67870-P) and the Generalitat de Catalunya (2014 SGR 137) (Spain) 
Author Contributions: The chemistry was carried out by S.A.H.A.M., Y.E.J, G.A.H.A. and the microbiology by E.J.R. Libraries were designed and supervised by A.E.-F., F.A., and B.G.d.l.T. The interpretation of results and discussion were carried out by all authors. The first drafts of the manuscript were prepared by S.A.H.A.M. and the final version included input from all authors.

Conflicts of Interest: The authors declare not conflicts of interest

\section{References}

1. Hamilton WL, Wenlock R. Antimicrobial resistance: A major threat to public health. Cambridge Med. J. 2016.

2. Ramesh, S.; Govender, T.; Kruger, H. G.; de la Torre, B. G.; Albericio, F., Short AntiMicrobial Peptides (SAMPs) as a class of extraordinary promising therapeutic agents. J. Peptide Sci. 2016, 22, 438-451.

3. Ling, L.L.; Schneider, T.; Peoples, A.J.; Spoering, A.L.; Engels, I.; Conlon, B.P.; Mueller, A.; Schäberle, T.F.; Hughes, D.E.; Epstein, S. A new antibiotic kills pathogens without detectable resistance. Nature 2015, 517, 455-459.

4. Jad, Y.E.; Acosta, G.A.; Naicker, T.; Ramtahal, M.; El-Faham, A.; Govender, T.; Kruger, H.G.; de la Torre, B.G.; Albericio, F. Synthesis and biological evaluation of a teixobactin analogue. Org. Lett. 2015, 17, 61826185.

5. Abdel Monaim, S.A.; Jad, Y.E.; Ramchuran, E.J.; El-Faham, A.; Govender, T.; Kruger, H.G.; de la Torre, B.G.; Albericio, F. Lysine scanning of Arg10-teixobactin: Deciphering the role of hydrophobic and hydrophilic residues. ACS Omega 2016, 1, 1262-1265.

(C) 2017 by the authors. Licensee MDPI, Basel, Switzerland. This article is an open access article distributed under the terms and conditions of the Creative Commons Attribution (CC BY) license (http://creativecommons.org/licenses/by/4.0/). 